Revta brasil. Bot., São Paulo,V.24, n.4 (suplemento), p.519-525, dez. 2001

\title{
Relações hídricas em espécies lenhosas no campus Pampulha/UFMG, Belo Horizonte, MG
}

\author{
BRUNO GUSMÃO SILVA ${ }^{1,2}$ e JOSÉ PIRES DE LEMOS FILHO ${ }^{1,3}$
}

(recebido: 5 de setembro de 2000; aceito: 5 de maio de 2001)

\begin{abstract}
Water relations of woody species in the campus Pampulha/UFMG, Belo Horizonte, MG). The present work aimed to evaluate the water relations as a function of the air evaporative demand and of the wood density in Swietenia macrophylla King, Joannesia princeps Vell., Inga edulis Mart., Licania tomentosa (Benth.) Fritsch and Centrolobium tomentosum Guill. ex Benth. The water potential was determined with a pressure chamber and the stomatal conductance with a diffusion porometer, in the rainy and dry seasons and in transitional situations. $J$. princeps presented higher values of water potential in predawn $\left(\psi_{\mathrm{PD}}>-0.25 \mathrm{MPa}\right)$, and I. edulis the smallest $\left(\psi_{\mathrm{PD}}=-1.5 \mathrm{MPa}\right)$. J. princeps showed higher water potential values during the day $\left(\psi_{\mathrm{MD}}>-1.5 \mathrm{MPa}\right)$ and lower values were observed for S. macrophylla and I. edulis $\left(\psi_{\mathrm{MD}}<-\right.$ 3.0 MPa). The daily amplitudes of the water potential $\left(\Delta \psi=\psi_{\mathrm{PD}}-\psi_{\mathrm{MD}}\right)$ were positively related with the vapour pressure deficit (VPD), and the largest values were observed in S. macrophylla and I. edulis. The conductance usually decreased in the beginning of the afternoon, and I. edulis presented smaller restriction to the transpiration. The largest conductance values were observed in a cloudy day of the rainy season. A quadratic regression $\left(r^{2}=0.635, p<0.01\right)$, suggests that species with larger density not necessarily present larger $\Delta \psi$, once a great restriction to the loss of water can happen as observed for $L$. tomentosa, a species with high wood density.
\end{abstract}

RESUMO - (Relações hídricas em espécies lenhosas no campus Pampulha/UFMG, Belo Horizonte, MG). O presente trabalho objetivou avaliar as relações hídricas em função da demanda evaporativa e da densidade da madeira em Swietenia macrophylla King, Joannesia princeps Vell., Inga edulis Mart., Licania tomentosa (Benth.) Fritsch e Centrolobium tomentosum Guill. ex Benth. O potencial hídrico foi determinado com uma câmara de pressão e a condutância estomática, com porômetro de difusão na estação chuvosa, na estação seca e nas situações transicionais. J. princeps foi a espécie que apresentou maiores valores de potencial hídrico no "predawn" $\left(\psi_{\mathrm{PD}}>-0,25 \mathrm{Mpa}\right)$, e I. edulis os menores valores $\left(\psi_{\mathrm{PD}}=-1,5 \mathrm{MPa}\right)$. J. princeps apresentou maiores valores de potencial hídrico durante o dia $\left(\psi_{\mathrm{MD}}>-1,5 \mathrm{MPa}\right)$, e os menores valores foram observados em $S$. macrophylla e I. edulis $\left(\psi_{\mathrm{MD}}<-3,0 \mathrm{MPa}\right)$. As amplitudes diárias do potencial hídrico $\left(\Delta \psi=\psi_{\mathrm{MD}}-\psi_{\mathrm{PD}}\right)$ foram relacionadas positivamente com o déficit de pressão de vapor (DPV) e os maiores valores foram observados em S. macrophylla e I. edulis. Geralmente a condutância diminuiu no início da tarde e I. edulis apresentou menor restrição à transpiração. Os maiores valores de condutância foram observados em um dia nublado na estação chuvosa, com baixo DPV. Uma regressão quadrática $\left(\mathrm{r}^{2}=0,635\right.$, $\mathrm{p}<0,01)$ sugere que, não necessariamente, as espécies com maior densidade apresentam maior $\Delta \psi$, uma vez que pode ocorrer uma grande restrição à perda d'água como verificado em $L$. tomentosa, a espécie com madeira mais densa.

Key words - Water potential, conductance, vapour pressure deficit, wood density

\section{Introdução}

Apesar do esforço de pesquisa a partir de trabalhos pioneiros como os de Ferri (1944) e Rawitscher (1948), ainda são limitados os estudo sobre relações hídricas em espécies lenhosas nativas do Brasil. A maioria dos estudos se concentra em espécies do cerrado e foi revisada

1. Universidade Federal de Minas Gerais, Campus da Pampulha, Instituto de Ciências Biológicas, Departamento de Botânica, 31270-110 Belo Horizonte, MG, Brasil.

2. Bolsista do programa PIBIC/FAPEMIG

3. Autor para correspondência: lemos@mono.icb.ufmg.br por Moraes \& Prado (1998) e por Franco (2000), com poucas informações disponíveis para espécies da amazônia (Roberts et al. 1990) e para espécies lenhosas da mata Atlântica (Alvim \& Alvim 1978, Lemos Filho \& Mendonça Filho 2000).

Trabalhando com espécies arbóreas na Costa Rica, Borchert (1994) concluiu que as alterações sazonais no "status" hídrico estão relacionadas com a disponibilidade de água no solo e com fatores bióticos como a profundidade e extensão das raízes, a densidade da madeira e a capacidade de armazenamento de água no caule. Tem sido considerado que uma grande quantidade de água armazenada, relatada como inversamente proporcional à densidade da madeira (Borchert 
1994), pode compensar o incremento da resistência hidráulica axial, com o aumento do tamanho da árvore, tendo um importante papel na regulação do "status hídrico" em situações de elevada demanda evaporativa (Goldstein et al. 1998).

Estudos recentes sobre a regulação do uso da água com espécies do cerrado (Franco 2000), sugerem que, a despeito do potencial acesso à água do solo devido às raízes profundas, a elevada demanda evaporativa do ar e as limitações hidráulicas associadas à obtenção de água na maior profundidade resultam em uma severa limitação estomática na transpiração. Estudando três espécies de leguminosas arbóreas da mata Atlântica, Lemos Filho \& Mendonça Filho (2000), também apontaram que as variações diárias do potencial hídrico $(\psi)$ têm relação com o déficit de pressão de vapor do ar (DPV), não ocorrendo limitações de água na rizosfera.

Considerando a importância, tanto do DPV como da capacidade de armazenamento de água no caule, nas relações hídricas, o presente trabalho objetivou determinar o comportamento do y e da condutância estomática em cinco espécies arbóreas, em função da demanda evaporativa do ar e da densidade da madeira, contribuindo para o entendimento das relações hídricas em espécies lenhosas tropicais.

\section{Material e métodos}

Foram estudadas as espécies: Swietenia macrophylla King, Joannesia princeps Vell., Inga edulis Mart., Licania tomentosa (Benth.) Fritsch e Centrolobium tomentosum Guill. ex Benth., as quais estão caracterizadas na tabela 1 quanto à fenologia foliar e densidade aparente da madeira (Lorenzi 1992, 1998). Todas as espécies encontram-se no Campus da UFMG, onde foram realizadas as medições. Para tal, foram escolhidas três plantas adultas de cada espécie, localizadas próximas entre si, para facilitar a tomada dos dados e minimizar o efeito ambiental. Na proximidade das plantas escolhidas foi instalado, à sombra, um psicrômetro para a obtenção do valor do déficit de pressão de vapor do ar. A variação diária na densidade de fluxo de fótons (DFF) foi determinada utilizando um sensor quântico Li-COR modelo LI 190.

Na base da copa de cada planta marcada, foram coletadas as folhas para a determinação do potencial hídrico com uma câmara de pressão segundo Scholander et al. (1965), antes do nascer do sol ("predawn") e no período de maior demanda evaporativa do ar, utilizando uma folha em cada planta, o mesmo procedimento empregado por Lemos Filho \& Mendonça Filho (2000). A condutância estomática foi
Tabela 1. Densidade da madeira e fenologia foliar de cinco espécies estudadas no Campus UFMG.

\begin{tabular}{lcc}
\hline Espécie & $\begin{array}{c}\text { Densidade } \\
\text { da madeira } \\
\left(\mathrm{g} \cdot \mathrm{cm}^{-3}\right)\end{array}$ & $\begin{array}{c}\text { Fenologia } \\
\text { foliar }\end{array}$ \\
\hline Swietenia macrophylla & $0,63^{\mathrm{a}}$ & semi-decídua \\
Joannesia princeps & $0,52^{\mathrm{a}}$ & semi-decídua \\
Inga edulis & $0,76^{\mathrm{b}}$ & sempre verde \\
Licania tomentosa & $0,98^{\mathrm{a}}$ & sempre verde \\
Centrolobium tomentosum & $0,75^{\mathrm{a}}$ & semi-decídua \\
\hline a conforme Lorenzi (1992) & & \\
b conforme Lorenzi (1998) & &
\end{tabular}

determinada com porômetro de difusão Delta T modelo AP4, na epiderme abaxial de duas folhas de cada uma das três plantas, nos diferentes horários. Os dados foram obtidos em plena estação seca (21/8/98 e 27/7/99), na estação chuvosa (23/1/ 99 e 11/11/99), e em situações transicionais (30/10/98, 24/5/ 99 e 12/4/2000), conforme pode ser verificado na figura 1 .

A partir do valor do potencial hídrico no "predawn" $\left(\psi_{\mathrm{PD}}\right)$ e dos valores mínimos registrados à tarde $\left(\psi_{\mathrm{MD}}\right)$ foram obtidos os valores das amplitudes de variação diária $\left(\Delta \psi=\psi_{\mathrm{PD}}-\psi_{\mathrm{MD}}\right)$, que foram relacionados com o déficit de pressão de vapor do ar (DPV) e com a densidade da madeira.

\section{Resultados e Discussão}

O máximo valor do déficit de pressão de vapor do ar (DPV), conforme pode ser verificado na tabela 2, foi registrado em 21/8/98, na estação seca (figura 1). Valores igualmente elevados foram registrados durante um veranico em $23 / 1 / 00$, dia em que se registrou a maior densidade do fluxo de fótons (DFF), acima de $2000 \mu \mathrm{mol} . \mathrm{m}^{-2} . \mathrm{s}^{-1}$ ao meio dia.

Em geral, da mesma forma que para espécies do cerrado (Franco 2000), e da mata atlântica (Lemos Filho \& Mendonça Filho 2000), os valores do $\psi_{\mathrm{PD}}$ nas espécies estudadas foram elevados (tabela 3). O menor valor do $\psi_{\mathrm{PD}}$ foi registrado para I. edulis, na estação seca $\left(\psi_{\mathrm{PD}}=-1,5 \mathrm{MPa}\right)$, e $J$. princeps foi a que apresentou os maiores valores $\left(\psi_{\mathrm{PD}}>-0,3 \mathrm{MPa}\right)$ (tabela 3 ). Os valores de potencial hídrico no "predawn" correspondem 


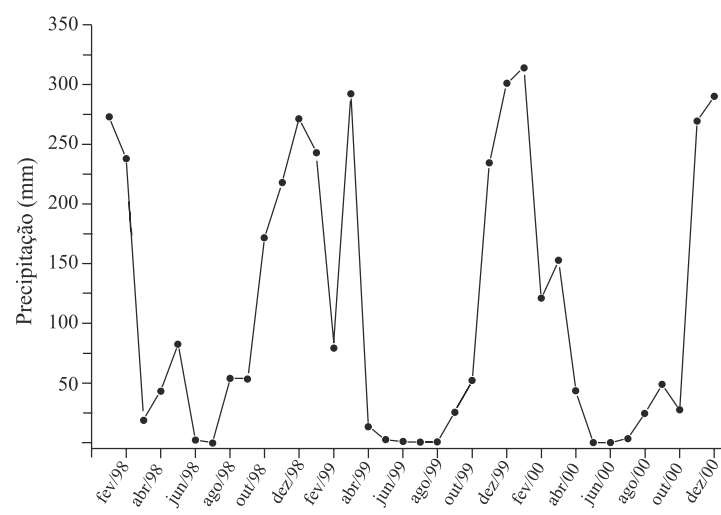

Figura 1. Totais pluviométricos mensais durante o período dos estudos (1998 a 2000), no Aeroporto da Pampulha, Belo Horizonte, MG.

aproximadamente ao potencial hídrico do solo (Andrade et al. 1998), uma vez que durante a noite, conforme relata Schimidhalter (1997), o potencial hídrico da planta tende a se equilibrar com o potencial hídrico das zonas úmidas do solo. Dessa forma, os elevados valores do $\psi_{\mathrm{PD}}$ na totalidade das espécies, no presente estudo, indicam a não ocorrência de limitação severa de água no solo, mesmo no final da estação seca.

No decorrer do dia os menores valores do potencial hídrico $\left(\psi_{\mathrm{MD}}\right)$ foram observados na estação seca, nas plantas de $S$. macrophylla e $I$. edulis $\left(\psi_{\mathrm{MD}}<-3,0 \mathrm{MPa}\right)$ e os maiores valores foram obtidos em $J$. princeps $\left(\psi_{\mathrm{MD}}>-1,2 \mathrm{MPa}\right)$. L. tomentosa e C. tomentosum apresentaram valores próximos a - 2,0 MPa. Em conseqüência do comportamento diferencial, os menores valores de amplitude diária do potencial hídrico $(\Delta \psi)$ foram observados para $J$. princeps, e os maiores para S. macrophylla e I. edulis. Esses resultados são equivalentes aos observados por Lemos Filho \& Mendonça Filho (2000) que registraram valores mínimos diários de até - 3,8 MPa em leguminosas lenhosas da mata atlântica. São também próximos aos citados para plantas lenhosas do cerrado que, segundo Franco (2000), variam entre - 1,0 e - 3,0 $\mathrm{MPa}$ na estação chuvosa e - 2,0 e - 4,0 MPa na estação seca. Esse resultado sugere que, como descrito para as espécies lenhosas do cerrado e da mata atlântica, as espécies do presente estudo apresentam decréscimo no potencial hídrico em resposta à demanda evaporativa do ar, a despeito da não limitação hídrica no solo (altos valores de $\left.\psi_{\mathrm{PD}}\right)$. Esse fato pode ser comprovado a partir dos dados apresentados na tabela 4 onde se observa, em geral, um razoável ajuste linear entre os valores máximos diários de DPV do ar e os valores de $\Delta \psi$, sugerindo uma estreita relação entre essas variáveis, da mesma forma que observado por Lemos Filho \& Mendonça Filho (2000).

Para todas as espécies, os valores de condutância estomática apresentaram decréscimo nos horários de maior demanda evaporativa do ar (tabela 5). Inga edulis, além de apresentar os maiores valores de condutância, independentemente da estação do ano, foi a espécie que apresentou menor restrição, com reduções inferiores a $50 \%$, à tarde nos dias de maior DPV. De forma oposta, $L$. tomentosa e $C$. tomentosum foram as espécies que apresentaram maior restrição da transpiração à tarde, com redução da condutância em até $80 \%$. As outras espécies apresentaram um comportamento intermediário. Esses dados sugerem que estas espécies apresentaram um comportamento similar ao descrito para muitas espécies do cerrado, restringindo a condutância estomática nas situações

Tabela 2. Déficit de pressão de vapor do ar (DPV) e densidade do fluxo de fótons (DFF) registrado às 9:00, 12:00 e 15:00h, nos diferentes dias durante o período estudado no Campus Pampulha/UFMG.

\begin{tabular}{|c|c|c|c|c|c|c|}
\hline \multirow[t]{2}{*}{ Dia } & \multicolumn{3}{|c|}{$\mathrm{DPV}(\mathrm{kPa})$} & \multicolumn{3}{|c|}{$\operatorname{DFF}\left(\mu \mathrm{mol} \cdot \mathrm{m}^{-2} \cdot \mathrm{s}^{-1}\right)$} \\
\hline & 09:00 & $12: 00$ & $15: 00$ & 09:00 & $12: 00$ & $15: 00$ \\
\hline $21 / 8 / 1998$ & 1,38 & 2,25 & 2,37 & 1002 & 1671 & 1121 \\
\hline $30 / 10 / 1998$ & 0,18 & 0,70 & 0,70 & 243 & 371 & 522 \\
\hline 23/1/1999 & 1,31 & 2,15 & 2,80 & 1412 & 2136 & 1533 \\
\hline $24 / 5 / 1999$ & 1,01 & 1,41 & 0,97 & 321 & 471 & 150 \\
\hline 27/7/1999 & 0,82 & 2,11 & 1,38 & 979 & 1530 & 140 \\
\hline $11 / 11 / 1999$ & 0,23 & 0,99 & 0,73 & 631 & 1147 & 1220 \\
\hline $12 /[4 / 2000$ & 0,70 & 2,07 & 1,77 & 1122 & 1846 & 1392 \\
\hline
\end{tabular}


Tabela 3. Potencial hídrico, no "predawn” $\left(\psi_{\mathrm{PD}}\right)$, menor valor diário $\left(\psi_{\mathrm{MD}}\right)$ e amplitude de variação do potencial hídrico $(\Delta \psi)$, em cinco espécies estudadas no Campus UFMG.

\begin{tabular}{|c|c|c|c|}
\hline Dia & $\psi_{\mathrm{PD}}(\mathrm{MPa})$ & $\psi_{\mathrm{MD}}(\mathrm{MPa})$ & $\Delta \psi(\mathrm{MPa})$ \\
\hline \multicolumn{4}{|c|}{ Swietenia macrophylla } \\
\hline $\begin{array}{c}21 / 8 / 98 \\
30 / 10 / 98 \\
23 / 1 / 99 \\
24 / 5 / 99 \\
27 / 7 / 99 \\
11 / 11 / 99 \\
12 / 4 / 00\end{array}$ & $\begin{array}{l}-1,00 \pm 0,10 \\
-0,77 \pm 0,14 \\
-1,10 \pm 0,06 \\
-1,25 \pm 0,20 \\
-1,03 \pm 0,32 \\
-0,50 \pm 0,06 \\
-0,43 \pm 0,21\end{array}$ & $\begin{array}{l}-3,18 \pm 0,04 \\
-1,20 \pm 0,12 \\
-2,20 \pm 0,12 \\
-2,40 \pm 0,33 \\
-2,50 \pm 0,41 \\
-2,03 \pm 0,26 \\
-1,67 \pm 0,29\end{array}$ & $\begin{array}{l}2,18 \pm 0,08 \\
0,43 \pm 0,09 \\
1,10 \pm 0,09 \\
1,15 \pm 0,12 \\
1,47 \pm 0,55 \\
1,53 \pm 0,09 \\
1,23 \pm 0,12\end{array}$ \\
\hline \multicolumn{4}{|c|}{ Joannesia princepsylla } \\
\hline $\begin{array}{c}21 / 8 / 98 \\
30 / 10 / 98 \\
23 / 1 / 99 \\
24 / 5 / 99 \\
27 / 7 / 99 \\
11 / 11 / 99 \\
12 / 4 / 00\end{array}$ & $\begin{aligned}-0,23 & \pm 0,03 \\
-0,10 & \pm 0,00 \\
-0,20 & \pm 0,00 \\
-0,05 & \pm 0,00 \\
-0,05 & \pm 0,00 \\
-0,05 & \pm 0,00 \\
-0,05 & \pm 0,00\end{aligned}$ & $\begin{aligned}-1,13 & \pm 0,07 \\
-0,25 & \pm 0,05 \\
-0,87 & \pm 0,07 \\
-0,90 & \pm 0,08 \\
-0,35 & \pm 0,04 \\
-0,37 & \pm 0,07 \\
-0,57 & \pm 0,06\end{aligned}$ & $\begin{array}{l}0,90 \pm 0,03 \\
0,15 \pm 0,06 \\
0,67 \pm 0,07 \\
0,85 \pm 0,06 \\
0,30 \pm 0,21 \\
0,32 \pm 0,09 \\
0,52 \pm 0,03\end{array}$ \\
\hline $\begin{array}{c}\text { Inga edulis } \\
21 / 8 / 98 \\
30 / 10 / 98 \\
23 / 1 / 99 \\
24 / 5 / 99 \\
27 / 7 / 99 \\
11 / 11 / 99 \\
12 / 4 / 00\end{array}$ & $\begin{array}{r}-1,40 \pm 0,10 \\
-0,27 \pm 0,02 \\
-0,58 \pm 0,25 \\
-1,50 \pm 0,00 \\
-0,30 \pm 0,33 \\
-0,10 \pm 0,05 \\
-0,07 \pm 0,03\end{array}$ & $\begin{array}{l}-3,22 \pm 0,02 \\
-2,13 \pm 0,04 \\
-2,93 \pm 0,07 \\
-2,60 \pm 0,00 \\
-2,10 \pm 0,41 \\
-0,87 \pm 0,07 \\
-1,40 \pm 0,53\end{array}$ & $\begin{array}{l}1,82 \pm 0,09 \\
1,87 \pm 0,06 \\
2,36 \pm 0,10 \\
1,10 \pm 0,00 \\
1,80 \pm 0,17 \\
0,77 \pm 0,07 \\
1,33 \pm 0,19\end{array}$ \\
\hline $\begin{array}{c}\text { Licania tomento } \\
21 / 8 / 98 \\
30 / 10 / 98 \\
23 / 1 / 99 \\
24 / 5 / 99 \\
27 / 7 / 99 \\
11 / 11 / 99 \\
12 / 4 / 00\end{array}$ & $\begin{array}{r}-0,25 \pm 0,00 \\
-0,20 \pm 0,03 \\
-0,37 \pm 0,09 \\
-0,37 \pm 0,08 \\
-0,37 \pm 0,22 \\
-0,10 \pm 0,05 \\
-0,12 \pm 0,08\end{array}$ & $\begin{aligned}-2,10 & \pm 0,10 \\
-0,80 & \pm 0,10 \\
-1,73 & \pm 0,13 \\
-1,10 & \pm 0,08 \\
-1,45 & \pm 0,04 \\
-0,60 & \pm 0,12 \\
-1,87 & \pm 0,29\end{aligned}$ & $\begin{array}{l}1,85 \pm 0,04 \\
0,60 \pm 0,10 \\
1,37 \pm 0,22 \\
0,73 \pm 0,18 \\
1,08 \pm 0,19 \\
0,50 \pm 0,08 \\
1,75 \pm 0,20\end{array}$ \\
\hline \multicolumn{4}{|c|}{ Centrolobium tomentosum } \\
\hline $\begin{array}{c}21 / 8 / 98 \\
30 / 10 / 98 \\
23 / 1 / 99 \\
24 / 5 / 99 \\
27 / 7 / 99 \\
11 / 11 / 99 \\
12 / 4 / 00\end{array}$ & $\begin{aligned}-0,43 & \pm 0,09 \\
-0,13 & \pm 0,03 \\
-0,37 & \pm 0,03 \\
-0,60 & \pm 0,00 \\
-0,15 & \pm 0,04 \\
-0,07 & \pm 0,02 \\
-0,50 & \pm 0,00\end{aligned}$ & $\begin{aligned}-2,17 & \pm 0,93 \\
-0,87 & \pm 0,06 \\
-0,87 & \pm 0,13 \\
-1,70 & \pm 0,08 \\
-1,30 & \pm 0,08 \\
-0,97 & \pm 0,55 \\
-1,53 & \pm 0,42\end{aligned}$ & $\begin{array}{l}1,74 \pm 0,09 \\
0,73 \pm 0,07 \\
0,50 \pm 0,03 \\
1,10 \pm 0,06 \\
1,15 \pm 0,01 \\
0,90 \pm 0,53 \\
1,03 \pm 0,19\end{array}$ \\
\hline
\end{tabular}

Tabela 4. Equações do ajuste linear relacionando máximo déficit de pressão de vapor do ar (DPV) e a amplitude da variação diária do potencial hídrico $(\Delta \psi)$, na estação seca, em cinco espécies estudadas no Campus UFMG.

\begin{tabular}{lllll}
\hline \multicolumn{1}{c}{ Espécie } & \multicolumn{1}{c}{ Ajuste linear } & $\mathrm{r}$ & $\mathrm{P}$ & $\mathrm{n}$ \\
\hline Swietenia macrophylla & $\Delta \psi=0,49271 \mathrm{DPV}+0,55993$ & 0,591 & 0,004 & 21 \\
Joannesia princeps & $\Delta \psi=0,24049 \mathrm{DPV}+0,13921$ & 0,509 & 0,001 & 21 \\
Inga edulis & $\Delta \psi=0,39998 \mathrm{DPV}+0,94688$ & 0,472 & 0,030 & 21 \\
Licania tomentosa & $\Delta \psi=0,67334 \mathrm{DPV}+0,03791$ & 0,710 & $3,09.10^{-4}$ & 21 \\
Centrolobium tomentosum & $\Delta \psi=0,60384 \mathrm{DPV}+0,28769$ & 0,464 & 0,034 & 21 \\
\hline
\end{tabular}


de elevada demanda evaporativa, notadamente na estação seca, conforme relatam Moraes \& Prado (1998) e Lemos Filho (2000).

A espécie que apresentou menor restrição da transpiração, Inga edulis, apresentou os menores valores de $\psi_{\mathrm{PD}}$ e grande amplitude diária $(\Delta \psi)$. Além de ser uma espécie sempre-verde, $I$. edulis apresenta madeira com maior densidade quando comparada com $J$. princeps (tabela 1). Essa espécie, além ser semidecídua, tem madeira mais leve, sugerindo maior capacidade de armazenamento de água, e apresentou também significativa restrição da condutância em dias de elevada demanda evaporativa do ar, fatos que podem explicar os maiores valores do $\psi_{\mathrm{PD}}$ e os menores valores de $\Delta \psi$ mesmo durante a seca.

Tabela 5. Condutância estomática em diferentes horários, em cinco espécies estudadas no Campus UFMG.

\begin{tabular}{|c|c|c|c|}
\hline \multirow[t]{2}{*}{ Dia } & \multicolumn{3}{|c|}{ Condutância $\left(\mathrm{mmol} \cdot \mathrm{m}^{-2} \cdot \mathrm{s}^{-1}\right)$} \\
\hline & $8: 30-9: 30$ & $11: 30-12: 30$ & $14: 30-15: 30$ \\
\hline \multicolumn{4}{|c|}{ Swietenia macrophylla } \\
\hline $21 / 8 / 98$ & $234,5 \pm 40,7$ & $87,8 \pm 27,0$ & $47,2 \pm 16,6$ \\
\hline $30 / 10 / 98$ & $111,3 \pm 18,4$ & $88,8 \pm 18,4$ & $64,6 \pm 13,1$ \\
\hline 23/1/99 & $97,8 \pm 13,1$ & $97,3 \pm 6,7$ & $65,7 \pm 10,9$ \\
\hline $24 / 5 / 99$ & $124,8 \pm 25,6$ & $100,2 \pm 15,8$ & $39,0 \pm 7,7$ \\
\hline 27/7/99 & $118,7 \pm 30,7$ & $65,9 \pm 27,2$ & $49,9 \pm 19,6$ \\
\hline $11 / 11 / 99$ & $189,4 \pm 41,2$ & $288,2 \pm 99,8$ & $190,5 \pm 67,7$ \\
\hline $12 / 4 / 00$ & $110,7 \pm 19,6$ & $63,7 \pm 7,3$ & $40,0 \pm 6,2$ \\
\hline \multicolumn{4}{|c|}{ Joannesia princepsylla } \\
\hline $21 / 8 / 98$ & $120,5 \pm 28,9$ & $68,0 \pm 28,1$ & $20,1 \pm 6,1$ \\
\hline $30 / 10 / 98$ & $126,7 \pm 31,6$ & $116,1 \pm 25,2$ & $62,4 \pm 26,5$ \\
\hline $23 / 1 / 99$ & $136,3 \pm 51,1$ & $144,7 \pm 44,1$ & $31,0 \pm 4,8$ \\
\hline $24 / 5 / 99$ & $102,8 \pm 15,1$ & $74,0 \pm 8,7$ & $23,2 \pm 2,8$ \\
\hline $27 / 7 / 99$ & $128,4 \pm 38,6$ & $52,6 \pm 20,9$ & $17,6 \pm 2,6$ \\
\hline $11 / 11 / 99$ & $228,5 \pm 47,3$ & $241,2 \pm 75,2$ & $145,8 \pm 17,3$ \\
\hline $12 / 4 / 00$ & $99,0 \pm 18,2$ & $93,6 \pm 9,2$ & $\begin{array}{r}14 J, 0 \\
40,4 \pm 9,6\end{array}$ \\
\hline \multicolumn{4}{|l|}{ Inga edulis } \\
\hline $21 / 8 / 98$ & $303,0 \pm 80,6$ & $214,3 \pm 28,9$ & $168,7 \pm 34,7$ \\
\hline $30 / 10 / 98$ & $192,6 \pm 39,6$ & $244,8 \pm 49,4$ & $172,2 \pm 38,6$ \\
\hline $23 / 1 / 99$ & $219,7 \pm 30,2$ & $283,0 \pm 68,8$ & $153,8 \pm 18,2$ \\
\hline $24 / 5 / 99$ & $289,7 \pm 55,6$ & $209,3 \pm 26,7$ & $210,0 \pm 18,5$ \\
\hline 27/7/99 & $229,2 \pm 28,9$ & $113,3 \pm 12,1$ & $132,7 \pm 51,4$ \\
\hline $11 / 11 / 99$ & $344,0 \pm 53,1$ & $331,7 \pm 79,8$ & $223,1 \pm 82,4$ \\
\hline $12 / 4 / 00$ & $197,1 \pm 32,1$ & $252,4 \pm 51,2$ & $176,9 \pm 24,2$ \\
\hline \multicolumn{4}{|c|}{ Licania tomentosa } \\
\hline $21 / 8 / 98$ & $159,3 \pm 22,0$ & $88,5 \pm 18,9$ & $45,8 \pm 7,7$ \\
\hline $30 / 10 / 98$ & $125,7 \pm 21,1$ & $73,6 \pm 36,6$ & $18,1 \pm 2,8$ \\
\hline $23 / 1 / 99$ & $165,7 \pm 31,1$ & $125,2 \pm 26,0$ & $32,6 \pm 10,1$ \\
\hline $24 / 5 / 99$ & $123,7 \pm 29,7$ & $41,9 \pm 11,9$ & $20,0 \pm 6,1$ \\
\hline 27/7/99 & $128,2 \pm 14,0$ & $56,4 \pm 10,4$ & $11,8 \pm 5,0$ \\
\hline $11 / 11 / 99$ & $174,8 \pm 47,5$ & $300,0 \pm 56,7$ & $107,0 \pm 65,3$ \\
\hline $12 / 4 / 00$ & $248,8 \pm 31,0$ & $92,6 \pm 6,5$ & $26,4 \pm 2,8$ \\
\hline \multicolumn{4}{|c|}{ Centrolobium tomentosum } \\
\hline $21 / 8 / 98$ & $166,8 \pm 56,3$ & $93,2 \pm 23,7$ & $80,6 \pm 33,5$ \\
\hline $30 / 10 / 98$ & $170,2 \pm 45,0$ & $50,1 \pm 8,8$ & $47,1 \pm 27,1$ \\
\hline 23/1/99 & $207,8 \pm 42,8$ & $75,8 \pm 9,3$ & $26,1 \pm 4,1$ \\
\hline $24 / 5 / 99$ & $158,5 \pm 23,2$ & $132,0 \pm 5,2$ & $22,3 \pm 1,2$ \\
\hline 27/7/99 & $174,0 \pm 16,1$ & $80,1 \pm 12,4$ & $63,9 \pm 16,4$ \\
\hline $11 / 11 / 99$ & $495,8 \pm 41,0$ & $151,5 \pm 22,3$ & $116,9 \pm 7,0$ \\
\hline $12 / 4 / 00$ & $176,1 \pm 27,6$ & $154,3 \pm 14,9$ & $72,0 \pm 7,0$ \\
\hline
\end{tabular}


Uma regressão quadrática entre a densidade da madeira e $\Delta \psi$, com razoável valor do coeficiente de determinação (figura 2), sugere que, não necessariamente, as espécies que apresentam maior densidade da madeira têm maior $\Delta \psi$ nos dias com elevada demanda evaporativa, uma vez que pode ocorrer uma grande restrição à perda d'água pelo fechamento dos estômatos, como verificado em $L$. tomentosa, a espécie com madeira mais densa, limitando a transpiração e, dessa forma, impedindo um maior decréscimo nos valores do potencial hídrico.

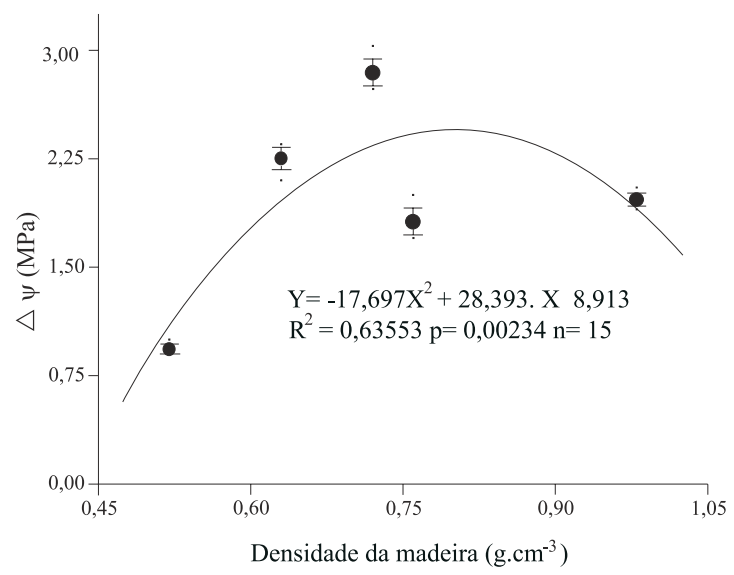

Figura 2. Relação entre a amplitude diária da variação do potencial hídrico $(\Delta \psi)$ em um dia da estação seca, com elevada demanda evaporativa e a densidade da madeira em cinco espécies estudadas no Campus UFMG.

No presente trabalho, realizado com árvores crescendo em ambiente urbanizado, sob maior impacto antrópico, os resultados, em geral, foram similares aos obtidos com espécies lenhosas em ambiente natural no Brasil, sugerindo o mesmo padrão de comportamento nas relações hídricas. O comportamento identificado para as espécies estudadas é similar ao descrito para espécies arbóreas do cerrado (Franco 2000), mostrando que, apesar da disponibilidade de água no solo, conforme sugerem os valores elevados de $\psi_{\mathrm{PD}}$, as limitações hidráulicas associadas à exploração da água em maior profundidade no solo, resultam em restrição da transpiração devido ao fechamento dos estômatos nos dias de elevada demanda evaporativa do ar.
Agradecimentos - Os autores agradecem o apoio recebido do CNPq (proc. 301255/92) e à FAPEMIG (Programa PIBIC), que possibilitaram a realização do presente trabalho.

\section{Referências bibliográficas}

ALVIM, P.T. \& ALVIM, R. 1978. Relation of climate to growth periodicity in tropical trees. In Tropical trees as living systems. (P.B. Tomlinson. \& M.H. Zimmermann, eds.) Cambridge University Press, Cambridge, p.445-464

ANDRADE, J.L., MEIZER, F.C., GOLDSTEIN, G., HOLBROOK, N.M., CAVELIER, J., JACKSON, D. \& SILVERA, K. 1998. Regulation of the water flux throughout trunks, branches and leaves in trees of a lowland tropical forest. Oecologia 115:463-471.

BORCHERT, R. 1994. Soil and stem water storage determine phenology and distribution of tropical dry forest trees. Ecology 75:1437-1449.

FERRI, M.G. 1944. Transpiração de plantas permanentes dos cerrados. Boletim da Faculdade de Ciências e Letras da Universidade de São Paulo, Botânica 4:161-224.

FRANCO, A.C. 2000. Water and light use strategies by cerrado wood plants. In Tópicos Atuais em Botânica: Palestras convidadas do $51^{\circ}$ Congresso Nacional de Botânica (T.B. Cavalcanti \& B.M.T. Walter, eds.). EMBRAPA-Recursos Genéticos e Sociedade Botânica do Brasil, Brasília, p.292-298.

GOLDSTEIN, G., ANDRADE, J.L., MEINZER, F.C.; HOLBROOK, N.M., CAVELIER, J., JACKSON, P. \& CELIS, A. 1998. Stem water storage and diurnal patterns of water use in tropical forest canopy trees. Plant, Cell and Environment 21:397-406.

LEMOS FILHO, J.P. 2000. Fotoinibição em três espécies do cerrado (Annona crassifolia, Eugenia dysenterica e Campomanesia adamantium) na estação seca e na chuvosa. Revista Brasileira de Botânica 23:45-50.

LEMOS FILHO, J.P. \& MENDONÇA FILHO, C.V. 2000. Seasonal changes in the water status of three wood legumes from the Atlantic forest, Caratinga, Brazil. Journal of Tropical Ecology 16:21-32.

LORENZI, H. 1992. Árvores brasileiras. Instituto Plantarum de Estudos da Flora, Nova Odessa, v.1.

LORENZI, H. 1998. Árvores brasileiras. Instituto Plantarum de Estudos da Flora, Nova Odessa, v.2.

MORAES, J.A.P.V., \& PRADO, C.H.B.A. 1998. Photosynthesis and water relations in Cerrado vegetation. In Ecophysiological strategies of xerophytic and amphibious plants in the Neotropics. (F.R. Scarano \& A.C. Franco, eds.). Oecologia brasiliensis. Instituto de Biologia, Universidade Federal do Rio de Janeiro, Rio de Janeiro, v. IV, p.45-63.

RAWITSCHER, F. 1948. The water economy of the vegetation of the "campos cerrados" in southern Brazil. Journal of Ecology 36:237-268.

ROBERTS, J., CABRAL, O.M.R. \& AGUIAR, L.F. 1990. Stomatal and boundary-layer conductances in an Amazonian Terra Firme forest. Journal of Applied Ecology 27:336-353. 
SCHOLANDER, P.F. HAMMEL, H.T. HEMINGSEN, E.A. \& BRADSTREET, E.D. 1965. Sap pressure in vascular plants. Science 148:339-346
SCHMIDHALTER, U. 1997. The gradient between pre-dawn rhizoplane and bulk soil matric potentials, and its relation to the pre-dawn root and leaf water potentials of four species. Plant, Cell and Environment 20:953-960. 
